\title{
MONDIALITÉ ET DIVERSITÉ CULTURELLE
}

\author{
OUMAR DIA
}

\begin{abstract}
This article is a plea for cultural diversity, not guaranteed by the two dominant and opposite forms governing the current process of globalization - abstract universalism and cultural sectarianism. While the former ignores our differences in the name of humanism, the latter proclaims to defend our identities but in fact leads to promoting self-closure and violence. Based on the work of Edouard Glissant, in particular on his concept of "globality", this article advocates a third way, which equally promotes cultural interpenetration and preservation of local identities. This third way represents both a realistic approach and a utopia. It cannot be achieved without significant effort and phased structural development - from the local to the global - with values stemming from cultural diversity. This article demonstrates that the fate of globality, and that is to say of cultural diversity, depends upon ethical choices every person makes.
\end{abstract}

\section{Introduction}

En intitulant ce texte Mondialité et diversité culturelle, nous avions pleinement conscience d'avoir peut-être laissé transparaître dès la lecture de l'intitulé ce que devrait être pour nous la mondialisation sur le plan culturel et plus précisément sur celui des rapports entre les différentes cultures de notre monde et de notre époque. Le choix du terme mondialité, en lieu et place de celui de mondialisation plus couramment usité n'est pas innocent. Il est en réalité révélateur d'une autre vision du processus en cours de la mondialisation (fortement marqué par une tendance à l'uniformisation) qu'il est souhaitable et nécessaire de réorienter pour mettre chaque culture - que l'on définit comme ce qui dans l'humain ne relève pas de la nature, qui n'est donc pas intangible parce que relevant de l'ordre de l'acquis et non

https://doi.org/10.14712/24646504.2020.5

(C) 2020 The Author. This is an open-access article distributed under the terms of the

Creative Commons Attribution License (http://creativecommons.org/licenses/by/4.0). 
de l'inné ${ }^{1}$ - dans les meilleures dispositions à s'ouvrir à des éléments extérieurs, à accueillir et à faire place à la différence tout en ne s'effaçant pas comme identité culturelle spécifique mais ouverte dans cette entrée en relation. Cette approche, déjà théorisée et vulgarisée par Édouard Glissant, ${ }^{2}$ considère l'interpénétration des cultures comme le meilleur moyen de faire advenir l'universel ${ }^{3}$. Elle s'oppose pour cette raison à la fois à l'universalisme abstrait qui dilue toutes les différences dans un tout indifférencié et au repli sectaire des cultures sur elles-mêmes qui peut conduire à ce que redoutait Amin Maalouf et qu'il désignait par l'expression effrayante d' 'identités meurtrières $»^{4}$.

Cette mondialité, qui valorise l'identité relation au détriment de l'identité uniforme et de l'identité sectaire repliée sur elle-même, peut être présentée comme une troisième voie et en tant que telle comme un recours face aux deux tendances radicalement opposées de l'actuelle mondialisation. La première de ces tendances, faut-il le rappeler, renvoie à un processus croissant d'homogénéisation de toutes les cultures et donc d'effacement des différences alors que la deuxième tendance consiste en un repli sectaire sur soi. ${ }^{5}$ Contre cette forme contrastée de la mondia-

1 La culture, relevant de la contingence, s'oppose à la nature qui relève de la nécessité. C'est sur cette idée d'une genèse et d'un développement des cultures à partir de situations contingentes, donc différentes et changeantes d'un endroit à un autre ou d'une époque à une autre, que se fonde le plaidoyer fait dans cet article en faveur de la diversité culturelle. La diversité culturelle, caractéristique de l'humanité, n'est donc pas le résultat de natures intangibles, immuables et fermées entre elles mais plutôt celui de situations contingentes, diverses et variées dans lesquelles naissent et se développent les différentes cultures.

2 Voir son Traité du Tout-monde, Paris, Gallimard, 1997, qui oppose la mondialité à la mondialisation, le Tout-monde qu'il définit comme le monde du voisinage et de la contamination mutuelle entre humains, paysages, cultures et spiritualités au Tout-empire qui dilue toutes les différences dans un Tout informe et indifférencié. Le mérite de l'alternative adoptée par Glissant dans ce livre, c'est qu'elle permet de conjurer les dangers du Tout-empire par la réalisation effective et consciente du Tout-monde.

3 Déjà, chez Senghor, l'universel était pensé en termes de rencontre et de dialogue des cultures et non en termes d'effacement des différences (cf. Senghor Léopold Sédar, "Le dialogue des cultures » in Liberté V, Paris, Éditions du Seuil, 1993).

4 Dans les Identités meurtrières, Paris, Grasset, 1992, Amin Maalouf, constatant la grande difficulté des gens à assumer des identités multiples dans des sociétés où ils n’ont pas tous la même langue, la même culture, la même religion, la même couleur de peau, etc., lance un appel à une autre pratique de l'identité pour prévenir d'éventuels conflits meurtriers.

5 Dans le texte qui a servi d'orientation à son séminaire doctoral de philosophie contemporaine pendant près d'une décennie à l'Université Cheikh Anta Diop de Dakar et intitulé « Projet d'une philosophie de la mondialisation » in Éthiopiques, n64-65, 1er et 2eme semestre 2000, Sémou Pathé Guèye admet la difficulté à définir de façon précise le concept de mondialisation et impute cela au caractère multidimensionnel et complexe du processus dont elle rend compte. S'appuyant sur ce caractère à la fois multidimensionnel et complexe, il est allé jusqu'à identifier douze tendances dans le processus actuel de la mondialisation. Ce sont essentiellement deux de ces douze tendances (la tendance à l'homogénéisation, voire à l'occidentalisation du monde et celle inverse et opposée du 
lisation, qui ne nous laisse le choix qu'entre le renoncement à notre identité ou le repli sectaire sur nous-mêmes, Édouard Glissant oppose ce qu'il assimile à « une pensée dynamique échevelée du Tout, [...] une haute pensée du monde » qu'il appelle «non pas la mondialisation (c'en est même le contraire) mais la mondialité " ${ }^{6}$; une pensée qu'il définit dans une référence explicite à Gilles Deleuze comme la Totalité-Monde qui engendre un monde du voisinage, de la contamination mutuelle entre les humains, leurs cultures et leurs spiritualités. Ce concept de mondialité, ainsi présenté par celui dont la pensée a été qualifiée à juste titre d'archipélique, peut être considéré comme un puissant rempart contre les deux plus grandes menaces de la mondialisation tantôt évoquées ${ }^{7}$. En tant que tel, il peut servir de cadre théorique pour une application effective de la Convention de l'UNESCO de 2005 sur la protection et la promotion de la diversité des expressions culturelles et la prééminence des principes de celle-ci - qui font de l'être humain une fin en soi - sur les directives de l'OMC. ${ }^{8}$ Son étude conceptuelle montre qu'il est, autant une donnée du monde (son avènement est inscrit dans l'histoire du monde) qu'une utopie. En tant qu'utopie (le monde peut être gros à la fois de la mondialité culturelle où les différentes cultures s'interpénètrent et s'enrichissent mutuellement et de ses deux contraires), l'avènement de la mondialité culturelle nécessite donc un combat et un engagement des femmes et des hommes qui croient en ses valeurs.

\section{La mondialité culturelle : une donnée du monde}

La mondialité culturelle, aperçu en a déjà été donné dans l'introduction, se caractérise par une interpénétration et un enrichissement mutuel des différentes cultures du monde. Comme telle, elle fait droit à l'authentique diversité qui

repli identitaire sur soi) que nous avons retenues pour définir la forme de mondialisation à laquelle s'oppose la mondialité.

6 Glissant E., La cohée du Lamentin. Poétique V, Paris, Gallimard, 2005, pp. 22-23.

7 Dans son ouvrage Un humanisme de la diversité. Essai sur la décolonisation des identités, Paris, GF, 2009, Alain Renaut dessine, à la suite d'Édouard Glissant, une troisième voie entre l'arrogance de l'universalisme abstrait qui consiste en un effacement pur et simple des différences et les excès du particularisme sectaire ou identités-racines.

8 Pointant du doigt, toujours dans le même ouvrage, l'orientation prise par la mondialisation économique, Alain Renaut attire l'attention sur les dangers qu' elle fait peser de nos jours sur toute forme de diversité. Il écrit à ce propos, ibid., pp. 287-288 : « [...] il s'agit dorénavant de s’alerter de la mise en péril mondiale d'une diversité culturelle dont l'effacement qui la menace renvoie moins au pouvoir de certains sur d'autres qu'à une dynamique plus vaste, plus souterraine aussi. Cette dynamique conduit les individus aussi bien que les sociétés à se détacher progressivement, par la logique d'un développement économique imposant ses conditions, des liens culturels qu'ils avaient hérités ou choisis ". 
consacre toujours le respect de l'autre dans son altérité. En faisant de la diversité culturelle son principe ou son fondement, elle préserve l'humanité non seulement de l'homogénéisation culturelle du monde qui se traduit par une suppression de toutes les différences, mais aussi du repli autarcique sur sa propre identité par peur ou rejet de l'autre. La mondialité culturelle consacre l'avènement d'un monde où, comme l'écrit Glissant, "on entre dans des zones de voisinage, plutôt qu'on n'acquiert des caractères formels $»^{9}$.

Pourtant, aussi paradoxal que cela puisse paraitre, c'est la mondialisation économique qui, en visant l'homogénéisation culturelle du monde, a créé les conditions de l'enracinement planétaire de la diversité. Certes, sous ses aspects les plus visibles et les plus dominants, la mondialisation donne de solides raisons de penser que l'uniformisation culturelle du monde est inéluctable. Mais, l'envisager sous ce seul point de vue pourrait être réducteur dans la mesure où cela reviendrait à faire l'impasse sur toutes les autres possibilités qu' elle recèlerait et dont la possible réalisation ne peut pas être totalement exclue. Au-delà de son aspect le plus visible, la mondialisation fournit également d'autres raisons (certes moins massives et moins apparentes que celles en faveur de l'uniformisation culturelle ou des replis identitaires violents) qui autorisent à la lire ou à l'interpréter comme une occasion sans précédent de faire advenir pour le meilleur de notre monde le règne de la diversité culturelle. En conséquence de quoi, elle ne devrait plus être considérée uniquement comme une menace, mais également comme une chance, fut-elle mince, pour l'avènement pacifique de la diversité culturelle. Empruntant à Hegel son concept de ruse de la raison, nous pourrions pousser l'audace plus loin en la considérant comme un moyen peut-être impropre dont se servirait l'Esprit du monde pour l'amener à faire advenir de façon inconsciente l'autre d'elle-même, à savoir la mondialité ${ }^{10}$.

Nous savons que, pour des raisons évidentes d'efficacité et de rentabilité économique, la mondialisation a pour visée fondamentale l'uniformisation culturelle du monde qui n'est rien d'autre qu'une dilution de toutes les identités particulières dans un tout uniforme. Toutefois, le procédé dont se sert la mondialisation pour atteindre cet objectif, à savoir l'ouverture grâce aux moyens de communication des différentes cultures les unes sur les autres, a aussi créé les conditions d'un dialogue planétaire entre les différentes entités culturelles, mettant du coup en échec

9 Glissant E., La cohée..., op. cit., p. 136.

10 Dans La raison dans l'histoire, trad. K. Papaoainnou, Paris, UGE, 1965, p. 106, Hegel, examinant les passions et sacrifices constatées dans l'histoire les présente comme des moyens dont se servirait l'Esprit du monde pour se protéger des passions un peu comme, lorsque nous voulons nous protéger contre les forces de la nature, nous les utilisons pour bâtir des maisons et nous mettre à l'abri. 
l'irréversibilité programmée par le marché de l'uniformisation culturelle. Rétrospectivement et de façon moins attendue, nous pourrions assister à un décalage entre la visée initiale de la mondialisation (l'uniformisation culturelle du monde) et la conséquence potentiellement inhérente à son propre développement (l'inéluctable diversité culturelle du monde). L'objectif conscient de fragilisation des différentes identités culturelles par la mondialisation s'accompagne paradoxalement d'une ouverture sans précédent des différentes cultures les unes sur les autres, préfigurant ainsi l'avènement de la mondialité culturelle en lieu et place de l'uniformisation qui était peut-être la plus attendue. Certes, le processus d'ouverture des différentes cultures les unes sur les autres est un processus aussi vieux que le monde. Mais, faut-il le reconnaître, il a connu son coup d'accélérateur le plus notable grâce à la mondialisation et à ses grandes avancées scientifiques et technologiques ${ }^{11}$. La conscience universelle d'habiter une seule terre ne s'est faite jour qu'à l'époque de la mondialisation et de ses moyens modernes de communication. En mettant en relation, grâce à la révolution des transports et aux autoroutes de l'information, les différents endroits de la terre, la mondialisation a réussi la prouesse d'amener des cultures, qui se croyaient jusque-là uniques, à la conscience qu'il en existe d'autres, certes différentes, mais non moins dignes du même statut. L'ouverture à la différence qui résulte inéluctablement de ce processus de découverte et de connaissance mutuelles est peut-être la meilleure garantie d'un dialogue pacifique entre les différentes cultures. Ce qu'Édouard Glissant a donc appelé il y a quelques années mondialité était manifestement quelque chose d'inévitable pour au moins deux raisons.

La première raison tient au fait que la planète terre sur laquelle nous vivons n'est pas infinie. Ce que les Nouvelles Technologies de l'Information et de la Communication (NTIC) ont réalisé d'extraordinaire c'est d'arriver à transformer notre monde en un espace fini ou, pour parler comme Marshall McLuhan, en un « village planétaire $»^{12}$. La réalité de la mondialisation est bien celle d'un monde fini, cerné de part en part par les satellites et par internet. C'est d'ailleurs la prise de conscience profonde d'une telle réalité qui a conduit Paul Virilio à définir à juste titre la mondialisation comme «la clôture du champ des possibles de l'horizon terrestre $»^{13}$.

11 Dans sa phase embryonnaire, la mondialisation a été favorisé par les grandes navigations occidentales, le colonialisme européen et la découverte d'un moyen de communication comme le télégraphe. Dans sa phase actuelle ou achevée, elle a comme moteur le développement de moyens de transport comme l'avion et l'essor des NTIC.

12 McLuhan Marshall, Pour comprendre les médias : les prolongements technologiques de l'homme, Paris, Seuil, 1997.

13 Virilio Paul, La bombe informatique, Paris, Galilée, 1995, p. 23. 
Comme cela a toujours été le cas pour de tels changements majeurs, l'idée de la nouvelle réalité de notre monde a précédé logiquement et historiquement son effectivité. Au début du dix-neuvième siècle, dans un contexte de guerres napoléoniennes pour la diffusion dans une Europe sans frontières des idéaux de liberté et d'égalité de la Révolution française, le philosophe de l'histoire Hegel avait avancé une idée prémonitoire, matérialisée d'une certaine façon aujourd'hui par le phénomène de la mondialisation. Avec son idée d'un monde unifié ${ }^{14}$ sur le plan politique par Napoléon et sur le plan philosophique par son propre système qui aurait acquis par la même occasion le statut de philosophie du monde ${ }^{15}$, Hegel a, malgré son adhésion évidente à l'impérialisme et à l'eurocentrisme, été de ceux qui ont anticipé le phénomène actuel de la mondialisation. En effet, grâce aux grandes avancées scientifiques et technologiques de l'ère de la mondialisation, des femmes et des hommes de divers horizons géographiques et culturels ont fini par se rencontrer et feront peut-être advenir ce que Senghor désirait et appelait de tous ses vœux par le concept de civilisation de l'universel et qui d'après lui serait l'œuvre de toutes les civilisations ${ }^{16}$. D'expansion en extension, des groupes, des clans et des tribus ont fini par se réunir dans des communautés élargies dont la complexité nécessitait la mise en place d'une organisation permettant de vivre ensemble dans la diversité. C'est ce mouvement ascensionnel de convergence d'identités plurielles qui s'est réalisé dans une unité consciente d'elle-même et qui préfigure ce que nous avons appelé avec Édouard Glissant la mondialité opposée au mondialisme en ce qu'elle n'oublie pas et ne supprime pas sa diversité initiale dans l'unité réalisée. En fait, le Toutmonde de la mondialité n'a rien d'une totalité indifférenciée, fermée et statique. Sa différence fondamentale avec cette dernière c'est que "son tout est un devenir », c'est-à-dire selon les mots de Glissant une totalité reflétant « la quantité réalisée de toutes les différences du monde, sans que la plus incertaine d'entre elles puisse en

14 Exposée sous forme de caution philosophique de l'impérialisme et de l'eurocentrisme, l'idée hégélienne d'un monde destiné à s'unifier se trouve dans une lettre du philosophe à son ami Niethammer du 13 octobre 1806, in Correspondance, trad. J. Carrère, tome I, pp. 114-115 où il écrit : « j' ai vu l'empereur cette âme du monde sortir de la ville pour aller en reconnaissance, c'est effectivement une sensation merveilleuse de voir un pareil individu qui, concentré ici sur un point, assis sur un cheval, s'étend sur le monde et le domine».

15 Voir " Philosophie du monde », in Magazine littéraire, n²93, novembre 1991 et Éternité et historicité de l'esprit selon Hegel, chap. III : « Politique et philosophie », Paris, Vrin, 1991 de Bernard Bourgeois.

16 Dans Liberté 1 : Négritude et humanisme, Paris, Seuil, 1964, Senghor fait le plaidoyer d'un universel qui serait le résultat d'un dialogue pacifique des cultures. Fondamentalement ouvert à la diversité, il s'oppose au modèle universaliste hégélien qui, en réduisant l'universel à la seule identité occidentale, nie de fait la diversité culturelle. Si la critique hégélienne de l'absolu de Schelling dans la préface à la Phénoménologie de l'esprit peut inciter à penser que Hegel adhère à une forme d'universel ouvert à la diversité, ses exposés de l'histoire de la philosophie et de la philosophie de l'histoire prouvent qu'il n'en est rien : pour lui l'universel a une identité singulière qui est celle du seul Occident. 
être distraite ${ }^{17}$. Le Tout-monde glissantien ne dilue pas les différences dans un absolu informe et sans mouvement mais les conserve et les enrichit dans l'unité réalisée. Une certaine proximité existe donc entre le concept de mondialité tel que conçu par Édouard Glissant et celui de la civilisation de l'universel de Senghor.

Dans un autre contexte historique, où l'esprit du temps commandait aux penseurs du monde noir de travailler à la réhabilitation des valeurs de celui-ci après plusieurs siècles de négation, Senghor avait présenté son concept de civilisation de l'universel comme une invitation à transcender les différences non pas dans le sens de leur effacement dans un universalisme indifférenciant et négateur comme celui de l'Occident des Lumières qui a été compatible avec le colonialisme mais d'un dialogue fécond entre toutes les cultures. En tant que « rendez-vous du donner et du recevoir ", la civilisation de l'universel de Senghor consacre une forme de symbiose entre les différentes cultures du monde, amenant les humains qui s'identifient à elles à se penser comme "divers, mais semblables, ou semblables, mais divers $»^{18}$.

La deuxième raison en faveur d'une convergence probable des différentes cultures vers un certain lieu et donc en faveur de la mondialité culturelle est à chercher dans la dynamique propre à chacune d'entre elles. S'intéressant dans son ouvrage précédemment cité à la dynamique propre aux différentes cultures, Alain Renaut avance l'idée selon laquelle elles seraient toutes traversées par une dialectique constante faite d'enracinement d'un côté et d'ouverture de l'autre. C'est dans ce sens qu'il écrit :

il est important de noter que les cultures, en tant que sédimentation du processus de culture comme arrachement à la nature, sont par définition le lieu d'une tension entre la liberté (l'écart à la nature), dont elles procèdent, et la quasi nature qu'en un sens elles risquent de constituer en se figeant et en se transformant sous une forme «naturalisée ». ${ }^{19}$

Aucune culture ne saurait donc vivre et se perpétuer en mettant exclusivement en avant une seule de ces deux facettes. L'enracinement est certes une condition nécessaire à la perpétuation d'une culture mais également insuffisante. Pour se perpétuer, une culture a non seulement besoin de s'enraciner mais aussi de s'ouvrir à des éléments extérieurs, d'entrer en relation avec d'autres cultures différentes.

17 Glissant E., La terre le feu l'eau et les vents. Une anthologie de la poésie du Tout-monde, Galaade, 2010, p. 19.

18 Cette expression est d'Alain Renaut, Un humanisme..., op. cit., p. 251.

19 Ibid., p. 257. 
Une culture vivante possède donc nécessairement une double face : une face de fermeture et d'absolu (enracinement) d'un côté et une face de rencontres et d'échanges (ouverture) de l'autre. C'est cette dialectique inhérente à la dynamique des cultures qui les conduit nécessairement à entrer en relation, à se rencontrer sans toutefois disparaître en tant qu'identités en devenir. Le dialogue interculturel est une donnée constante de la dynamique des cultures. S'il est avéré que ses formes varient (elles peuvent être violentes ou pacifiques), il reste constant qu'il reste le meilleur atout dont disposeraient les différentes cultures pour conjurer d'éventuels déclins et continuer à vivre. L'idée d'une culture qui serait complètement et définitivement repliée sur elle-même, qui se serait développée en dehors de toute forme de métissage et de rencontres comme celle qualifiant la civilisation grecque de miraculeuse, est une simple vue de l'esprit. Dans les faits, une culture est beaucoup plus complexe que cela : n'étant pas une identité permanente et sans mouvement, elle se nourrit forcément d'emprunts extérieurs. C'est dans ce sens que Cheikh Anta Diop, en guise de mise au point à ceux qui seraient tentés d'interpréter sa thèse de l'unité culturelle de l'Afrique noire comme conférant aux cultures africaines des caractères permanents, statiques et fermés sur eux-mêmes, écrit dans Civilisation ou barbarie : «[...] tous les traits spécifiques des sociétés africaines analysées dans l'Unité culturelle de l'Afrique Noire n'ont rien de permanent ; il s'agit de traits profonds, mais non figés à jamais $»^{20}$. Au-delà de la culture africaine, ce diagnostic de Cheikh Anta Diop s'applique en réalité à toutes les cultures du monde qui, par leurs propres dynamiques, feront advenir la mondialité culturelle qui constitue la meilleure réponse au défi culturel que leur a posé la mondialisation et que le philosophe sénégalais Sémou Pathé Guèye, empruntant la formule à Senghor, a présenté comme le défi consistant à " assimiler sans être assimilé »"${ }^{21}$. Loin de conduire à une fusion des cultures, la relation interculturelle préserve donc plutôt l'identité continuellement enrichie de chaque culture sans pour autant la condamner à se replier sur elle-même. Toutefois prévient Glissant, pour que la relation interculturelle ne conduise pas fatalement à une fusion des cultures, il est indispensable que subsiste entre ces dernières un minimum d'opacité22 amenant Alain Renaut commentant le théoricien de la mondialité à écrire : «À cette condition seule, qui impose que le monde de la Relation puisse 'consentir à l'opacité se peut 'accomplir' véritablement, à travers le divers, l'humain'. »23

\footnotetext{
Diop Cheikh Anta, Civilisation ou barbarie, Paris, Présence africaine, 1981, p. 458.

21 Guèye Sémou Pathé, « Projet d'une philosophie de la mondialisation », in Éthiopiques, n $64-65$, 1er et 2ème semestre 2000, p. 188.

22 Glissant E., Poétique de la relation, Paris, Gallimard, 1990, p. 197.

23 Renaut A., Un humanisme..., op. cit., p. 336.
} 
Mais l'analyse qui vient d'être faite du processus hautement probable d'avènement de la mondialité culturelle doit-elle pour autant dispenser de toute lutte et de tout militantisme en faveur d'un monde où la diversité culturelle serait une réalité ? Le fait que le monde soit en réalité gros de mondialité culturelle et donc de diversité doit-il amener à sous-estimer ou à négliger les risques d'avortement du nouveau monde dont est grosse notre époque?

\section{La nécessaire mobilisation en faveur du combat pour la diversité culturelle}

En novembre 2001, l'Organisation des Nations Unies pour l'Éducation, la Science et la Culture (UNESCO) adoptait, à l'occasion de la $31^{\mathrm{ème}}$ session de sa Conférence générale, la Déclaration universelle sur la diversité culturelle. En inscrivant le droit de chacun à l'identité culturelle parmi les droits humains inaliénables, cette déclaration comblait de fait ce qui était manifestement un grave manquement dans la Déclaration universelle des droits de l'homme et du citoyen de 1948. C’est donc naturellement qu'elle a été accueillie par les défenseurs de la diversité culturelle comme une étape importante, à l'orée du troisième millénaire en vue d'humaniser la mondialisation jusque-là dominée par sa dimension marchande.

Dans la Préface à l'ouvrage du philosophe congolais Charles Zacharie Bowao intitulé La Mondialité: entre histoire et devenir, Souleymane Bachir Diagne écrit ceci: " on a en effet raison d'appeler à la résistance face à la globalisation qui ne sait penser la totalité que si celle-ci se fait marché où tout est marchandise. ${ }^{24}$. La mondialité, se définissant comme l'inverse de la mondialisation - «(le) revers névrotique de la (mondialité) écrit Glissant, résume dans le réel tout ce que nous appelons mondialisation »-, assigne comme tâche de résister à différents niveaux à cette dernière parce qu'elle oriente dangereusement notre monde vers un néant absolu de standardisation et de marchandisation de tous les produits et activités humaines. Considérant la diversité culturelle comme un obstacle de taille à l'efficacité économique et à la rentabilité financière érigées en dogmes, la mondialisation promeut, avec une certaine efficacité, l'uniformisation culturelle de notre monde sur la base des valeurs occidentales indûment et abusivement drapées du sceau de l'universalité. Dans L'Occident et les Autres. Histoire d'une suprématie 25 , l'historienne des rapports Nord-Sud Sophie Bessis décrit avec beaucoup de lucidité

24 Bowao Charles Zacharie, La mondialité entre histoire et devenir, Paris, Éditions Paari, 2004, p. 11.

25 Bessis Sophie, L'Occident et les autres. Histoire d’une suprématie, Paris, La Découverte, 2001. 
et d'objectivité l'histoire du processus de « confiscation de l'universel » par une identité particulière qui est en l'occurrence l'identité occidentale. D'où la nécessité selon Alain Renaut de lier le processus historique en cours de promotion de la diversité à celui d'une nécessaire décolonisation des identités. Contre cette logique de réduction de l'universel à une identité particulière qui est au moins depuis le dix-huitième siècle l'identité occidentale, il est impératif de rechercher et de promouvoir de nouvelles valeurs fondées sur le droit inaliénable à la diversité culturelle devant être reconnu et ratifié par l'ensemble des pays membres de l'Organisation des Nations Unies (O.N.U). C'est seulement de cette façon que nous donnerons un nouveau sens à nos existences et que nous contribuerons de façon décisive à la préservation de ce qui fait ultimement notre humanité : la diversité culturelle. Dans le combat légitime contre la totalisation et l'uniformisation en cours de la mondialisation économique, le concept de mondialité d'Édouard Glissant s'offre à nous comme un puissant viatique. Opposant, contrairement à l'opinion commune qui les confond, les concepts de Tout-Monde et de mondialisation, Édouard Glissant dira que la mondialité, qui ne laisse en rade aucune partie du monde ni aucune culture, requiert que soit reconnue l'exigence du divers. C'est dans ce sens qu'il écrit : "si la Trinidad et le Québec n'existaient pas comme composantes acceptées du Divers, il manquerait quelque chose à la chair du monde $»{ }^{26}$ Travailler à ne pas dépouiller le monde de sa substance revient à faire sien le postulat selon lequel il n'existe aucune essence identitaire permanente, mais des complexités radicalement mosaïques qui se nourrissent et s'enrichissent entre elles. Une telle prise de conscience constitue une étape incontournable dans la lutte contre la totalisation et l'uniformisation culturelle en cours de la mondialisation économique.

Dans La cohée du Lamentin, Édouard Glissant place la poétique de la Relation au cœur de son entreprise de promotion de la diversité culturelle, l'amenant ainsi à écrire : « [...] le Tout-monde s'apprend ou s'estime en premier lieu par une poétique de l'Utopie née d'une pratique de nos totalités, que nous appellerions mondialité. $»^{27}$ Pour Glissant donc, le Tout-monde possède une double facette. Il est à la fois une réalité concrète et une Fiction. C'est en ce sens qu'il est, d'après lui, assimilable au monde que nous avons tourné dans notre pensée et qui nous tourne en retour dans son roulis ${ }^{28}$. Fiction et réalité concrète, la mondialité est un concept éthique, une expérience active et solidaire du monde contemporain qui demande à prendre conscience de ses formes. Compte tenu de ce fait, Glissant juge aujourd'hui nécessaire de repenser et de régénérer en la divisant l'idée d'utopie

26 Glissant E., Le Discours antillais, Paris, Seuil, 1981, p. 407.

27 Glissant E., La cohée..., op. cit., p. 143.

28 Glissant E., Tout-monde, Paris, Gallimard, Folio, 1993, p. 208. 
dont le statut actuel diffère de celui qu'elle avait par le passé. Traditionnellement, l'idée d'utopie était fondamentalement soucieuse de perfection formelle et était liée aux pensées de système selon un principe d'économie de l'inutile. Récusant le caractère immuable de la conception classique de l'utopie, Glissant propose une alternative adaptant ce concept à la réalité processive et informe du monde contemporain où la prévue est préférée à la prévision qui elle serait liée au sens périmé de la causalité. C'est ainsi qu'à l'utopie occidentale qui « dessine une forme parfaite et l'enjoint à une réalité que l'esprit se donne pour fin de réformer ${ }^{29}$, Glissant oppose celles qu'il considère comme les utopies du futur, plus ouvertes parce qu' elles " ne se fonderont pas sur des présupposés, ne se voudront pas des réformes » et mieux " intégreront la démesure " $^{30}$. S'appuyant sur des oppositions binaires qui mettent à jour les différences entre les utopies anciennes et celles du Tout-monde, Glissant opte sans ambigüité pour la démesure contre la mesure, l'accumulation contre l'élection, la totalité contre le totalitaire, l'absence de présupposés contre la soumission à des modèles, le refus de réformer contre la pulsion de réforme, l'inachèvement contre la pulsion de la forme parfaite. Une telle régénérescence de l'idée d'utopie va chez lui de pair avec une nouvelle entreprise de remobilisation des Humanités contemporaines en faveur de la diversité. Ce glissement se justifie par le fait que la mondialité, en tant que pensée élaborée du Tout-monde, donc en tant que simple prise de conscience des formes ou plutôt des processus du futur en gestation ne suffit pas à faire advenir un monde de la diversité culturelle. Pour que celui-ci soit une réalité, il faudrait que cette pensée élaborée du Tout-monde, que cette connaissance ne se réduise pas à une simple analyse conceptuelle ou à un diagnostic froid. Une mobilisation en faveur de la mondialité requiert au préalable que cette pensée ou cette connaissance agisse sur les femmes et les hommes d'aujourd'hui avec l'énergie des mythes. C'est manifestement une telle fonction que revendique le discours novateur de Glissant. Mais quelle pourrait être la cible ou l'audience prioritaire de ce discours?

De notre point de vue, l'école et surtout l'université, où se décident de plus en plus les différentes trajectoires sociales et professionnelles de chaque génération, devraient être les cibles prioritaires de la diffusion de la nouvelle utopie et des mythes qu' elle aura engendrés. Pour qu'une telle démarche soit efficace, on peut par exemple inciter les 185 États ayant adhéré à la Déclaration de l'UNESCO de 2001 sur la diversité culturelle à orienter leurs systèmes éducatifs et universitaires dans le sens d'une meilleure réception par les jeunes générations de la philosophie

29 Glissant E., La cohée..., op. cit., p. 141.

30 Ibid., p. 142. 
et des valeurs de la diversité culturelle. Cette démarche des États devra être accompagnée par une adhésion et une implication des différents services publics et surtout des médias dans les stratégies de promotion de la diversité culturelle comme droit inaliénable de chaque être humain. Mais cela suffit-il pour convaincre tous aujourd'hui de la nécessité de faire siennes les valeurs de la diversité culturelle ? N'est-il pas en dernière instance du ressort de chaque individu dans son intériorité la plus insaisissable de choisir sa façon de se rapporter à la diversité culturelle ? Autrement dit, le choix éthique du sujet n'est-il pas ultimement le fondement de l'adhésion ou non aux valeurs de la diversité culturelle?

Dans son ouvrage déjà cité dans ce texte, Alain Renaut étend l'adoption de la diversité culturelle comme valeur humaine fondamentale à la sphère de l'éthique. Il écrit à ce propos :

il est vrai qu'un autre espace, celui de l'éthique, demeure ouvert pour que chacun se saisisse de l'exigence de procéder en la matière, celle de la diversité culturelle comme d'autres figures de la diversité, à des choix de valeurs induits par la déconstruction de l'universalisme assimilationniste et du différentialisme se fermant à l'universel. ${ }^{31}$

Le sort de la cause de la diversité culturelle ne se décidera donc pas exclusivement sur la base du niveau d'impact des politiques de sensibilisation menées par les pouvoirs publics et les médias sur les citoyens du monde. L'ultime choix dépend en réalité de chaque individu qui seul peut décider de quelle manière il compte bien se rapporter à la diversité culturelle.

Dans les Fondements de la métaphysique des mours, Emmanuel Kant, parlant de l'impératif catégorique sur lequel se fonde toute conduite authentiquement morale, le présente comme étant d'une part universel et d'autre part logiquement nécessaire. L'impératif catégorique est universel pour Kant parce qu'il vaut pour tous les cas et pour tous les hommes. Et il est nécessaire parce qu'il vaut par sa cohérence logique interne. Une maxime n'est donc un impératif catégorique que si elle est à la fois universelle et nécessaire. C'est du moins le sens que l'on peut tirer de ses deux principales formules qu'il énonce comme suit :

Agis uniquement d'après la maxime qui fait que tu peux vouloir en même temps qu' elle devienne une loi universelle. Agis de telle sorte que tu traites l'humanité aussi bien dans ta personne que dans la personne de tout autre toujours en même temps comme une fin, et jamais simplement comme un moyen. ${ }^{32}$

31 Renaut A., Un humanisme..., op. cit., p. 344.

32 Kant E., Fondements de la métaphysique des mours, trad. V. Delbos, Paris, Delagrave, 1969, p. 137. 
La première formule de cet énoncé de l'impératif catégorique met en avant le critère de l'universalité. Toutefois, cette exigence d'universalité n'est pas à confondre avec le proverbe ordinaire consistant à dire «ne fais pas à autrui ce que tu ne voudrais pas qu'il te fasse ». Un tel proverbe pose le principe de la réciprocité et revient de fait à un calcul d'intérêt : si je ne veux pas qu' autrui me cause du tort, alors je dois commencer par m'abstenir de lui en causer. Pour Kant, l'authentique universalité va bien au-delà : elle pose comme exigence d'une maxime qu' elle vaille pour l'humanité entière. Quant à la seconde formule de l'impératif catégorique, elle met en avant la valeur absolue de la personne. À l'opposé d'une chose qui, dépourvue de conscience et de raison, peut servir de moyen à l'homme (comme une pierre, un animal ou un outil), la personne est un être conscient, raisonnable et responsable. Elle ne peut donc être ramenée au rang d'un instrument.

En appliquant la première formule de cet impératif catégorique kantien à la question qui nous a occupé dans la présente réflexion, nous sommes amenés à considérer l'adhésion aux valeurs de la diversité culturelle comme la condition même d'existence et de pérennité du fait culturel. Rejeter le droit inaliénable à la diversité culturelle revient donc de fait à nier toute culture y compris celle à laquelle on s'identifie le plus. D'un point de vue strictement kantien, la diversité culturelle est logiquement nécessaire. Pour cette raison, elle ne peut être niée qu'au prix du rejet - même inconscient -, de sa propre identité culturelle. Quant à l'autre leçon, importante à notre époque, à tirer de la deuxième formule de l'impératif catégorique kantien, on peut tout simplement retenir qu' elle exclut toute logique marchande du champ de la diversité culturelle. Contre la mondialisation économique, qui fait du profit le seul universel indubitable et qui ne trouverait donc pour cette raison d'intérêt à promouvoir la diversité culturelle que dans la mesure où elle serait rentable, la deuxième formule de l'impératif catégorique de Kant permet quant à elle de soustraire la diversité culturelle de toute logique commerciale en la consacrant comme fin en soi qui ne saurait en aucun cas être réduite à un bien commercial qui serait susceptible d'être échangé contre de l'argent.

Que pouvons-nous retenir de ce détour par Kant dans la mise en place d'une pédagogie de promotion et d'adoption par tous de la diversité culturelle comme valeur humaine fondamentale? Fondamentalement, l'idée qu'il revient toujours en dernière instance à chaque individu, en son âme et conscience, de choisir d'adhérer ou non aux valeurs de la diversité culturelle. C'est cette posture ultimement éthique en chacun de nous que, au-delà de la question spécifiquement culturelle, Alain Renaut étend à celle de la diversité en général. C’est dans ce sens qu'il écrit : 
Le rapport à soi, ou, si l'on préfère, les obligations envers soi-même, de même que le rapport à l'autre ou aux autres, c'est-à-dire les obligations qui balisent ce rapport à autrui, constituent intrinsèquement l'espace de l'éthique. (...) L'éthique ne structure cependant le rapport aux autres que si, dans le rapport que chacun entretient avec luimême (donc dans son rapport à soi), celui-ci s'est déjà reconnu dans certains choix de valeurs, s'il les a en quelque sorte, au fil de sa vie et de la réflexion qui accompagne sa vie, fait siens. (...) Parce que, dans l'espace propre de l'éthique, c'est le sujet moral qui, au-delà de tels apprentissages, ne peut ultimement que s'obliger lui-même à souscrire aux principes éthiques de son existence, ce sujet même m'apparaît, dans le choix qu'il fait de sa vie, l'acteur indépassable qui décide de la place tenue dans sa vie par sa perception de la diversité humaine. ${ }^{33}$

\section{Conclusion}

Considérant, dans La cohée du Lamentin, la mondialité comme l'une des principales missions de notre temps, Édouard Glissant la décrit comme «[...] une aventure sans précédent qu'il nous est donné à tous de vivre, dans un espace-temps qui, pour la première fois, réellement et de manière foudroyante, se conçoit à la fois unique et multiple, et inextricable $»^{34}$. Si le rapport à ce phénomène de la diversité culturelle peut, comme nous l'avons vu plus tôt, différer d'un individu à un autre, il va de soi que plus personne aujourd'hui ne peut raisonnablement nier son évidence. D'ailleurs, les entreprises et les organisations internationales l'ont si bien compris qu'elles se conforment de plus en plus, dans leurs actions publiques, au principe de la diversité culturelle. C'est dans ce sens qu'une organisation internationale comme le Programme des Nations Unies pour le développement (PNUD) avait opportunément intitulé en 2004 son rapport mondial sur le développement humain qu'il produit annuellement « la liberté culturelle dans un monde diversifié " ${ }^{35}$. Au-delà du PNUD, cet intitulé traduit la prise de conscience de plus en plus grande que l'un des défis majeurs de notre époque consiste à organiser le phénomène de la diversité en mettant en place des structures qui permettent de vivre ensemble tout en étant différents sur le plan culturel. D’ailleurs, quand Édouard Glissant définit le Tout-monde comme " cette ouverture, de lieu en lieu, tous également légitimés, et chacun d'eux en vie et connexion avec tous les autres, et aucun

\footnotetext{
Renaut A., Un humanisme..., op. cit., pp. 426-427.

Glissant E., La cohée..., op. cit., p. 23.

Rapport mondial du PNUD sur le développement humain, 2004.
} 
d'eux réductible à quoique ce soit ${ }^{36}$, il ne fait rien d'autre que donner l'exacte mesure de ce nouveau défi qui peut se formuler ainsi : comment amener notre monde à devenir dans sa globalité plus juste à l'égard de la diversité culturelle ? Pour entrevoir une réponse à ce défi, un dernier détour par Édouard Glissant peut s'avérer utile. En tant que "poétique de la diversité solidaire ${ }^{37}$ », la mondialité porte en son sein un monde où

la relation entre les différents n'inaugure ni ne récapitule une géographie isolée, en tout cas pas une géographie seulement, mais une géographie assumée : puisque la différence du Tout-monde d'avec lui-même est qu'il est totalité non réalisée mais visible pourtant dans l'avenir. ${ }^{38}$

Le monde n'est peut-être donc pas seulement gros de diversité culturelle mais aussi probablement de l'essentiel des mécanismes qui permettront de gérer les défis que posera ce phénomène nouveau pour le plus grand nombre. Marx ne disait-il pas d'ailleurs à juste raison que l'humanité ne s'est jamais posée que des problèmes dont elle a la solution ${ }^{39}$ ?

Oumar Dia est enseignant-chercheur à l'Université Cheikh Anta Diop de Dakar. Ses enseignements portent sur la philosophie critique, l'idéalisme allemand et la philosophie de la diversité. Membre fondateur et secrétaire général de la Société sénégalaise de Philosophie (SO.SE.PHI), il a dirigé l'ouvrage collectif l'Afrique au cour de la mondialisation : hommage au professeur Sémou Pathé Guèye, Montréal, Presses panafricaines, 2016.

E-mail : oumar.dia@ucad.edu.sn

\footnotetext{
Glissant E., La cohée..., op. cit., pp. 136-137.

Ibid., p 143.

Glissant E., La terre..., op. cit., p 19.

Marx Karl, Contribution à la critique de l'économie politique, Paris, Éditions sociales, 1972, p. 4.
} 